\title{
Two species of Psocoptera (Insecta) as new records to the Bulgarian fauna with a country checklist
}

\author{
Dilian Georgiev
}

Department of Ecology and Environmental Conservation, University of Plovdiv, Tsar Assen Street 24, 4000 Plovdiv, Bulgaria, diliangeorgiev@abv.bg; https://orcid.org/0000-0003-2885-4895

\begin{abstract}
Two species of Psocoptera (Insecta) are reported as new to the Bulgarian fauna: Philotarsus parviceps (Philotarsidae) and Loensia fasciata (Psocidae). After this publication, the list of known barkfly species in the country contains a total of 67 species. A checklist is provided with references to the original records.
\end{abstract}

Keywords: Balkans, barkflies, distribution

\section{Introduction}

The insects from the order Psocoptera are very poorly studied in Bulgaria. Till the author began working on barkflies, only 29 species had been registered in the country (Popov 1939, 1961, Drensky, 1953, Lienhard 1998, Giese, 1964, Grigorov, 1975, Meinander, 1978, Lienhard \& Smithers, 2002, Tomov \& Trencheva, 2013, Sziráki 2013). Recently, the list was extended to 65 known species, most of which were summarised by Lienhard (2020). In this short communication, I report two more species as new records to the Bulgarian barkfly fauna.

\section{Material and methods}

Barkflies were collected by beating the vegetation in June and August 2020 by the author. Specimens were then stored in ethanol and after processing, deposited in the collection of the author. Species identifications and taxonomy follow Lienhard (1998).

\section{Results}

Philotarsidae

Philotarsus parviceps Roesler, 1954

Material examined: 6.8.2020, Rila Mountains, Borovets Resort, pine forest dominated by Picea abies, Abies alba and Pinus peuce, from dry brunches of pine trees with a lot of lichens, 5 q 9 , collected by beating the vegetation (Fig. 1b).

Psocidae

Loensia fasciata (Fabricius, 1787)

Material examined: 6.6.2020, Stara Planina Mountains, Uzana Area, west of Gabrovo Town, at the border of a meadow and a plantation of Picea abies, from dry brunches with a lot of lichens of unidentified dead deciduous tree, 2 우, collected by beating the vegetation (Fig. 1a). 


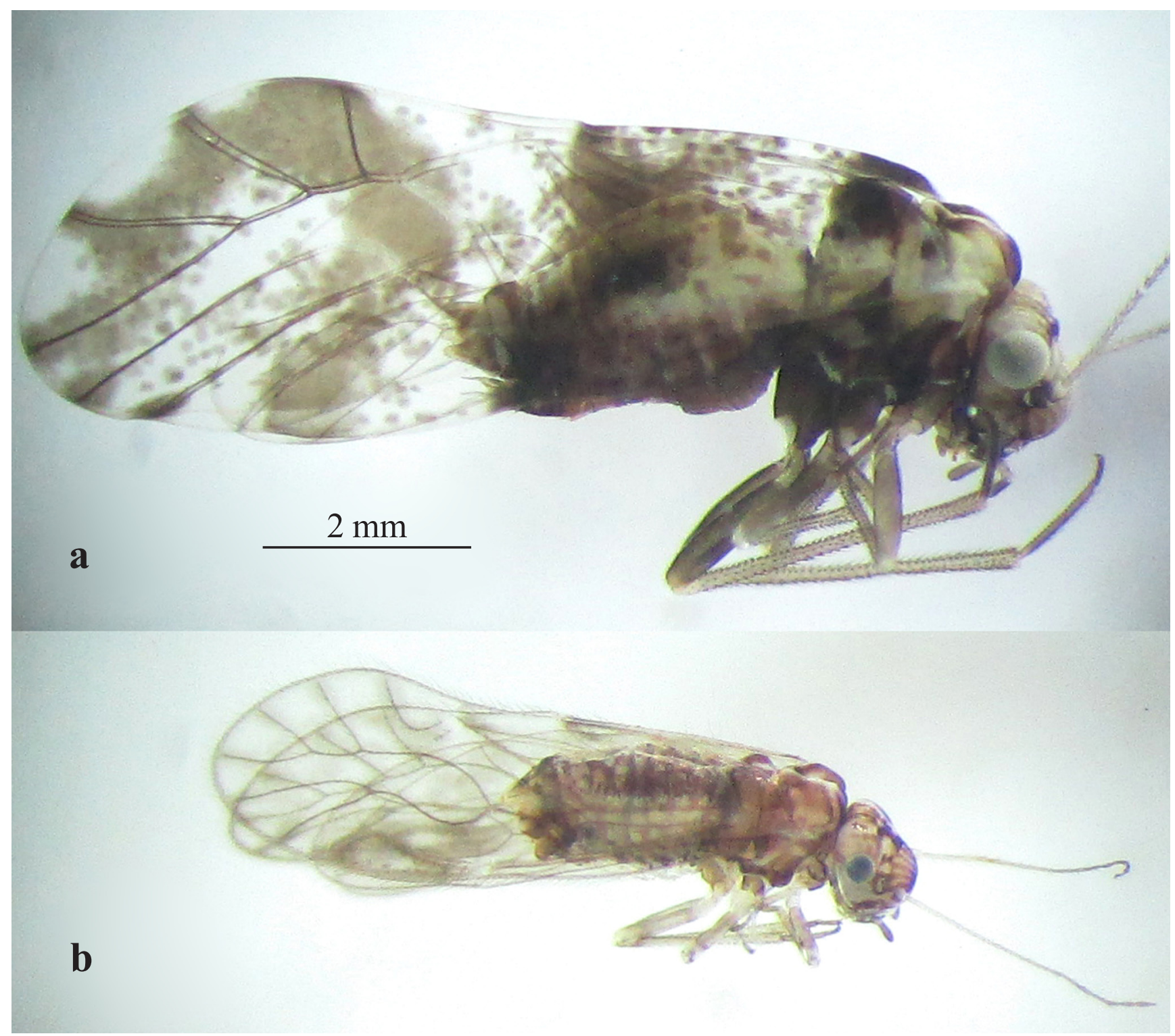

Fig. 1. (a) Female of Loensia fasciata (Fabricius, 1787) from Stara Planina Mts, Uzana Area; (b) female of Philotarsus parviceps Roesler, 1954 from Rila Mts, Borovets Resort.

Check list of the species of Psocoptera known in Bulgaria till 2020 (total of 67) with references to the original records:

\section{Trogiidae}

- Cerobasis guestfalica (Kolbe, 1880): Georgiev (2017b, 2018b), Georgiev \& Todorov (2018)

- Lepinotus reticulatus Enderlein, 1905: Georgiev (2016a, 2020), Georgiev \& Ivanova (2018), Georgiev \& Todorov (2018)

- Lepinotus inquilinus Heyden, 1850: Georgiev (2017b)
- Trogium pulsatorium (Linnaeus, 1758): Popov (1948, 1961), Drensky (1953), Giese (1964), Obretenchev et al. $(1993,1997)$

Psyllipsocidae

- Dorypteryx domestica (Smithers, 1958): Georgiev (2018b)

- Psyllipsocus ramburii Selys-Longchamps, 1872: Georgiev (2016b, 2018b, 2020), Georgiev \& Ivanova (2018) 
Prionoglarididae

- Prionoglaris stygia Enderlein, 1909: Georgiev \& Ivanova (2018), Georgiev (2019)

Liposcelididae

- Liposcelis bostrychophila Badonnel, 1931: Georgiev (2017b, 2018b, 2020)

- Liposcelis corrodens (Heymons, 1909): Popov (1939, 1961), Drensky (1940), Georgiev (2017d, 2020)

- Liposcelis decolor (Pearman, 1925): Drensky, (1940, 1953), Popov (1948), Giese (1964), Grigorov (1975), Georgiev (2017d, 2018b, 2020), Georgiev \& Todorov (2018)

- Liposcelis formicaria (Hagen, 1865): Giese (1964), Georgiev (2017b)

- Liposcelis meridionalis (Rosen, 1911): Georgiev (2017c)

- Liposcelis pearmani Lienhard, 1990: Georgiev (2017a, 2020), Georgiev \& Todorov (2018)

- Liposcelis priesneri Enderlein, 1925: Georgiev (2017a)

- Liposcelis rufa Broadhead, 1950: Georgiev (2017b)

- Liposcelis silvarum (Kolbe, 1888): Drensky (1953), Giese (1964), Georgiev (2017a, 2019)

Epipsocidae

- Bertkauia lucifuga (Rambur, 1842): Lienhard (1998), Georgiev (2017b, 2019, 2020)

Caeciliusidae

- Enderleinella obsoleta (Stephens, 1836): Giese (1964)

- Caecilius fuscopterus (Latreille, 1799): Georgiev \& Todorov (2017), Georgiev (2019)

- Valenzuela burmeisteri (Brauer, 1876): Giese (1964), Sziraki (2013), Georgiev \& Todorov (2018)

- Valenzuela despaxi (Badonnel, 1936): Giese (1964), Sziraki (2013)

- Valenzuela flavidus (Stephens, 1836): Giese (1964), Sziraki (2013), Georgiev (2019, 2020), Georgiev \& Todorov (2018), Georgiev \& Ivanova (2019)

- Valenzuela piceus (Kolbe, 1882): Drensky (1953), Sziraki (2013), Georgiev \& Todorov (2018), Georgiev (2020)
Stenopsocidae

- Graphopsocus cruciatus (Linnaeus, 1768): Drensky (1953), Giese (1964), Georgiev \& Todorov (2018), Georgiev (2019, 2020), Georgiev \& Ivanova (2019)

- Stenopsocus immaculatus (Stephens, 1836): Sziraki (2013), Georgiev (2017b)

- Stenopsocus lachlani Kolbe 1880: Giese (1964), Sziraki (2013)

Amphipsocidae

- Kolbia quisquiliarum Bertkau, 1882: Georgiev (2018a)

Lachesillida e

- Lachesilla pedicularia (Linnaeus, 1758): Drensky (1953), Giese (1964), Grigorov (1975), Georgiev \& Todorov (2018), Georgiev $(2019,2020)$

- Lachesilla bernardi Badonnel, 1938: Georgiev (2018a, 2020)

- Lachesilla quercus (Kolbe, 1880): Georgiev \& Todorov (2017), Georgiev (2020)

Ectopsocidae

- Ectopsocus briggsi McLachlan, 1899: Georgiev (2017b, 2020), Georgiev \& Ivanova (2019)

- Ectopsocus meridionalis Ribaga, 1904: Georgiev (2017b, 2020), Georgiev \& Ivanova (2019)

- Ectopsocus petersi Smithers, 1978: Georgiev (2020)

- Ectopsocus vachoni Badonnel, 1945: Georgiev (2017a)

Peripsocidae

- Peripsocus alboguttatus (Dalman, 1823): Sziraki (2013), Georgiev $(2019,2020)$

- Peripsocus didymus Roesler, 1939: Georgiev (2017b), Georgiev \& Todorov (2018)

- Peripsocus phaeopterus (Stephens, 1836): Popov (1948), Sziraki (2013), Georgiev \& Todorov (2018), Georgiev (2020)

- Peripsocus subfasciatus (Rambur, 1842): Sziraki (2013)

Trichopsocidae

- Trichopsocus dalii (McLachlan, 1867): Georgiev (2017b) 
Pseudocaeciliidae

- Trimerocaecilius cf. becheti Meinander, 1978: Georgiev \& Todorov (2018)

- Trimerocaecilius popovi Meinander, 1978: Meinander (1978), Georgiev (2017b)

Philotarsidae

- Aaroniella badonneli (Danks, 1950): Georgiev \& Todorov (2018)

- Philotarsus parviceps Roesler, 1954: present publication

- Philotarsus picicornis (Fabricius, 1793): Giese (1964), Sziraki (2013)

Elipsocidae

- Cuneopalpus cyanops (Rostock, 1876): Georgiev (2017a, 2019, 2020), Georgiev \& Todorov (2018)

- Elipsocus abdominalis Reuter, 1904: Georgiev (2018a)

- Elipsocus annulatus Roesler, 1954: Georgiev (2018a)

- Elipsocus hyalinus (Stephens, 1836): Georgiev \& Todorov (2018)

- Elipsocus moebiusi Tetens, 1891: Georgiev (2017a, 2019, 2020)

- Elipsocus pumilis (Hagen, 1861): Giese (1964), Georgiev (2020)

- Hemineura dispar Tetens, 1891: Giese (1964), Sziraki (2013), Georgiev (2020) - reported as "species complex"

Mesopsocida e

- Mesopsocus immunis (Stephens, 1836): Georgiev (2017a)

- Mesopsocus unipunctatus (Müller, 1764): Giese (1964), Georgiev (2020)

Psocidae

- Amphigerontia contaminata (Stephens, 1836): Georgiev (2017b, 2020)

- Blaste conspurcata (Rambur, 1842): Georgiev (2017b, 2020), Georgiev \& Todorov (2018)

- Loensia fasciata (Fabricius, 1787): present publication

- Loensia pearmani Kimmins, 1941: Giese (1964)

- Loensia variegata (Latreille, 1799): Georgiev \& Todorov (2018)

- Metylophorus nebulosus (Stephens, 1836): Giese (1964), Georgiev (2017b, 2020)
- Neopsocus rhenanus Kolbe, 1882: Lienhard (1998), Sziraki (2013), Georgiev (2020)

- Oreopsocus montanus (Kolbe, 1884): Giese (1964), Georgiev (2019)

- Psococerastis gibbosa (Sulzer, 1776): Giese (1964), Georgiev (2017b)

- Psocus bipunctatus (Linnaeus, 1761): Georgiev (2017b)

- Trichadenotecnum innuptum Betz, 1983: Georgiev (2017b)

- Trichadenotecnum majus (Kolbe, 1880): Georgiev (2017b)

- Trichadenotecnum sexpunctatum (Linnaeus, 1758): Giese (1964)

\section{Acknowledgments}

I am grateful to Dr Miroslav Antov (Faculty of Biology, Plovdiv University) for providing some of the literature sources.

\section{References}

Drensky P. 1940 Saobshtenie varhu vredni nasekomi. Izvestiya na Balgarskoto Entomologichno Druzhestvo 11: 249-253. (In Bulgarian)

Drensky P. 1953 Varhu razprostranenieto na razreda Copeognatha (Insecta) v Balgaria. Izvestiya na Zoologicheskiya Institut s Muzey 2: 377-381. (In Bulgarian)

Georgiev D. 2016a First record of Lepinotus reticulatus Enderlein, 1904 (Psocoptera: Trogiidae) from Bulgaria. Ecologica Montenegrina 9: 54-55.

Georgiev D. 2016b Psyllipsocus ramburii Selys-Longchamps, 1872 (Insecta: Psocoptera) from Andaka Cave - first record of the species from Bulgaria. Historia naturalis bulgarica 23: 179-181.

Georgiev D. 2017a New Barkfly records from Bulgaria (Insecta: Psocoptera). Klapalekiana 53: 7-9.

Georgiev D. 2017b Little known and newly recorded species of Psocoptera in Bulgaria. Ecologica Montenegrina 11: 74-79.

Georgiev D. 2017c Liposcelis meridionalis (Rosen, 1911) (Psocoptera: Liposcelididae) - new to the Bulgarian fauna. Ecologia Balkanica 9 (1): 103104.

Georgiev D. 2017d Two Species from the genus $\mathrm{Li}$ poscelis Motschulsky, 1852 - New to the Fauna 
of Bulgaria Found in the Building of the Natural History Museum - Plovdiv (Insecta: Psocoptera). Bulletin of the Natural History Museum - Plovdiv 2: 29-30.

Georgiev D. 2018a Four species of Psocoptera as new records for Bulgaria. ZooNotes 130: 1-2.

Georgiev D. 2018b Psocoptera records in the city of Plovdiv. Bulletin of the Natural History Museum - Plovdiv, 2018, Supplement 1: 47-48.

Georgiev D. 2019 A case study on the Barkfly fauna of Vrachanski Balkan Nature Park (Insecta: Psocoptera). ZooNotes, Supplement 7: 35-38.

Georgiev D. 2020 Study on the Psocoptera fauna of Sarnena Gora Mts. ZooNotes, Supplement 9: 8694.

Georgiev D., Ivanova V. 2018 Psocoptera records from caves of Bulgaria. Bulletin of the Natural History Museum - Plovdiv 3: 39-40.

Georgiev D., Ivanova V. 2019 First Psocoptera records from Northwest Bulgaria: a case study. ZooNotes 147: 1-2.

Georgiev D., Todorov O. 2017 Reports of Caecilius fuscopterus (Latreille, 1799) and Lachesilla quercus (Kolbe, 1880) in Bulgaria with some Additional Barkfly Records (Insecta: Psocoptera). Bulletin of the Natural History Museum - Plovdiv 2: 31-32.

Georgiev D., Todorov O. 2018 New information on the Psocoptera fauna of the Bulgarian Black Sea Coast. Bulletin of the Natural History Museum Plovdiv 3: 27-31.

Giese B. 1964 Ergebnisse der Albanien-Expedition 1961 des Deutschen Entomologischen Institutes. Beiträge zur Entomologie 14: 245-249.

Grigorov 1975 Nepriyateli po skladiranite produkti i materiali. Sofia, 177 pp. (In Bulgarian)

Lienhard C. 1998 Psocoptères euro-méditerranées. Faune de France 83: 1-517.
Lienhard C. 2020 Additions and corrections (part 19) to Lienhard \& Smithers, 2002: "Psocoptera (Insecta) - World catalogue and Bibliography". Psocid News 22: 16-29.

Lienhard C., Smithers C. 2002 Psocoptera (Insecta). World Catalogue and Bibliography. Muséum d'histoire naturelle, Genève, 745 pp.

Meinander M. 1978 Two new species of Pseudocaeciliidae (Psocoptera) from SE Europe. Entomologica Scandinavica 9: 1-6.

Obretenchev D., Tsvetkov D., Atanasov H., Obretencheva A. 1993 Ikonomicheski opasni nepriyateli po sahraniavanite bilki i drogi i borbata s tyah. Rastitelna Zashtita 10: 22-23. (In Bulgarian)

Obretenchev D., Tsvetkov D., Atanasov H., Obretencheva A. 1997 Vredni chlenestonogi (Arthropoda) po skladirani bilki, drogi i podpravki v Bulgaria. Acta entomologica bulgarica 3 (3-4): 33-39. (In Bulgarian)

Popov V. 1939 Skladovi nasekomi i borbata s tyah. Izdanie na Instituta za zashtita na rasteniyata $\mathrm{v}$ Sofia, 184 pp. (In Bulgarian)

Popov V. 1948 Nepriyateli po skladiranite produkti i materiali v Bulgaria i borbata s tyah. Nauchno populyarna poreditsa, BAN, 5, $414 \mathrm{pp}$.

Popov V. 1961 Skladovi nepriyateli i borbata s tyah. Zemizdat, Sofia, 199 pp. (In Bulgarian)

Sziráki G. 2013 Data to the Psocoptera fauna of Balkan Peninsula and two Aegean islands. Folia Historico-naturalia Musei Matraensis 37: 65-70.

Tomov R., Trencheva K. 2013 Annotated List of Pests (Insecta) on Stored Products, Reported in Bulgaria till 2012. Plant Science 50: 115-126. (In Bulgarian) 